\title{
Concepción y realización de celosías cerámicas, una evolución constructiva
}

\section{Ceramic lattices, design and developed, a construction evolution}

\author{
$\underline{\text { F. Pich-Aguilera }}^{(*)}$, T. Batlle ${ }^{(*)}, \underline{\text { P. Casaldàliga }}{ }^{(*)}$
}

\section{RESUMEN}

El presente artículo analiza diversas celosías cerámicas, realizadas por el equipo de arquitectura Pich-Aguilera en colaboración con diversas industrias, tratando de ilustrar particularidades en su comportamiento ambiental y su desarrollo tecnológico.

Mediante dicho análisis el trabajo trata de reflexionar sobre el sistema constructivo de la celosía cerámica como el resultado de la combinación de componentes y sistemas cerámicos muy diversos, implementados a lo largo del tiempo en distintas tipologías constructivas y funcionales. Paralelamente el estudio apunta algunas hipótesis acerca de las invariantes esenciales del propio sistema y de posibles evoluciones futuras.

Palabras clave: celosía cerámica; comportamiento ambiental; desarrollo tecnológico; producción industrial.

\section{ABSTRACT}

This paper analyzes several ceramic lattices, designed and developed by the architectural firm Picharchitects together with different industries. The paper tries to point out some of this construction system's particularities in relation with its environmental behavior and technological development.

At the same time the paper introduces some reflections about the lattices as a combination of different and diverse components that have been implemented along several construction technics and architectural functionalities. Moreover the paper tries to introduce some hypothesis about the construction system invariabilities (as an essences of the system itself) and some of its possible evolutions.

Keywords: ceramic lattices; environmental behavior; technological development; industrial production.

(*) Picharchitets, C/ Ávila, 138, 4r 1. ${ }^{\mathrm{a}} 08018$ Barcelona (España).

Persona de contacto/Corresponding author: info@picharchitects.com (Felipe Pich-Aguilera, Pau Casaldàliga)

Cómo citar este artículo/Citation: Pich-Aguilera, F., Batlle, T., Casaldàliga, P. (2016). Concepción y realización de celosías cerámicas, una evolución constructiva. Informes de la Construcción, 68(544): e170, doi: http://dx.doi.org/10.3989/ic.15.158.m15.

Copyright: (c) 2016 CSIC. Licencia / License: Salvo indicación contraria, todos los contenidos de la edición electrónica de Informes de la Construcción se distribuyen bajo una licencia de uso y distribución Creative Commons Attribution License (CC BY) Spain 3.o. 


\section{INTERÉS Y ALCANCE ESTUDIO}

El interés del presente análisis surge de la determinante necesidad en la arquitectura actual por poder reducir la demanda energética de los edificios en la consecución de su confort interior.

Las necesidades térmicas y lumínicas del interior de los edificios implica mayoritariamente el control de la incidencia solar sobre sus paramentos (1). La celosía cerámica actúa en este sentido como filtro que permite controlar ganancias solares y niveles de iluminación, a la vez que genera espacios intermedios entre el interior y el exterior de los edificios (2) (3).

A su vez un análisis tecnológico de la evolución de la celosía cerámica indica las posibilidades evolutivas de este sistema constructivo para adaptarse a los requerimientos de la arquitectura contemporánea.

Como síntesis de esa evolución tecnológica se apunta a ciertos estadios o canceles:

- Los sistemas tradicionales surgen directamente de la arquitectura vernácula en climas con altos niveles de radiación solar. Están basados en piezas cerámicas muy elementales y una colocación húmeda, a base de mano de obra intensiva de alta cualificación artesanal (4).

- A medida que las circunstancias edificatorias van demandando mayor flexibilidad y ligereza al paramento de celosía; la proporción y complejidad de la pieza cerámica tiende a aumentar, propiciando de este modo la invención e industrialización del sistema. Caracteriza esta evolución la incorporación de mecanismos complementarios de ensamblaje de las piezas cerámicas y una puesta en obra seca. Esto conlleva la ideación e implementación de nuevos modos de ejecución, alternativos a los oficios tradicionales.

- Posteriormente, la tecnificación de la celosía impulsa un salto desde el simple paramento a la membrana, alcanzando de ese modo una máxima flexibilidad, mínimo peso y mínima puesta en obra, a partir de un sistema altamente industrializado. Desde este estadio el sistema puede seguir

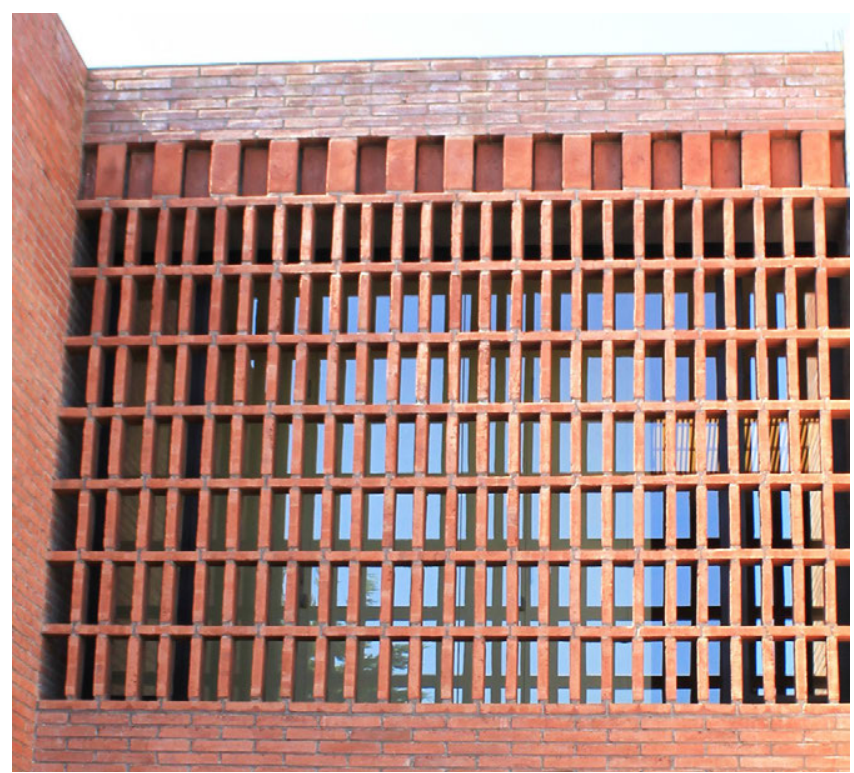

Figura 1. Vista exterior de la celosía. su evolución pivotando sobre el elemento cerámico, el cual irá acumulando complejidad para obtener siempre mejores respuestas térmicas, lumínicas, visuales, etc. (5).

- Finalmente, la necesaria transformación progresiva de la pieza cerámica, así como su carácter vertebrador, precisará de una industria fabricante altamente tecnificada, y tomando compromiso con la ideación, producción y colocación más cualitativa que cuantitativa, que lidere y garantice la pervivencia futura del sistema (6).

\section{REVISIÓN DE LAS OBRAS E HIPÓTESIS}

El estudio sintetiza el conocimiento acumulado en la evolución de celosías cerámicas a través de su aplicación en edificios reales del estudio Pich-Aguilera.

\subsection{Desde la tradición}

La arquitectura vernácula propia del mediterráneo ha desarrollado una rica tradición constructiva vinculada a la celosía cerámica. Este sistema está presente de un modo u otro en todos los arquetipos construidos por las diversas civilizaciones y configura la arquitectura más significativa de esa zona en su larguísima historia (7).

También podemos considerar la celosía como una respuesta tecnológica a las particularidades del clima, y el uso específico de cerámica aporta al sistema las propiedades concretas de la arcilla, dado que es un recurso abundante en sus diversas áreas geográficas.

La inmensa variedad formal y constructiva de las celosías y presentes en la tradición mediterránea nos transmite cómo las civilizaciones del pasado tenían un profundo conocimiento de las condiciones del clima y de cómo modularlo mediante el recurso de la arquitectura.

\subsubsection{Ejemplo A. Celosía cerámica para conjunto de} viviendas unifamiliares en Barcelona (1997)

En la zona de distribución de esta vivienda unifamiliar en Barcelona la disposición del filtro solar frente a un paramen-

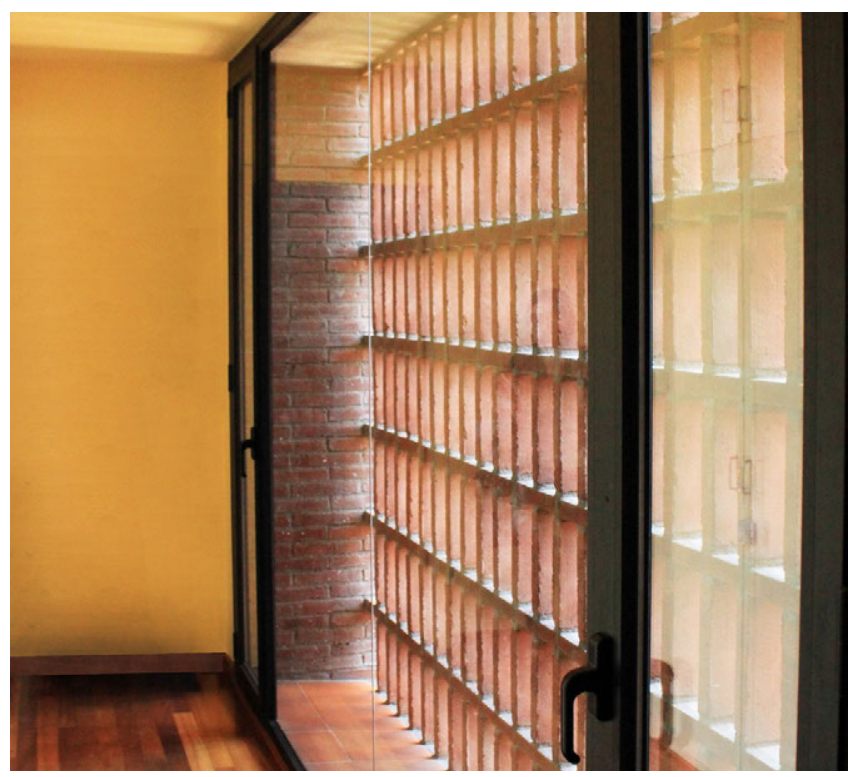

Figura 2. Vista interior de la celosía. 
to permite controlar la radiación solar incidente sobre el espacio interior. La creación de un espacio intermedio facilita una gradación térmica y la evacuación del calor.

A nivel tecnológico se trata de una versión actualizada de celosía tradicional, a base de ladrillos macizos tomados con mortero. El paramento se apoya sobre un elemento de soporte y se traba con el resto de paramentos. El paramento requiere cierto grosor para su estabilidad frente a los empujes horizontales del viento.

El montaje no sigue especificaciones técnicas definidas y depende de la pericia de la mano de obra.

\subsection{Más cerámica y menos peso}

A menudo los programas de uso contemporáneos obligan a flexibilizar el espacio interior de nuestros edificios incorporando soluciones estructurales de mayor complejidad, tales como vanos de gran amplitud y uniones isostáticas. La estructura deja entonces de ser un elemento rígido y homogéneo donde apoyar y confinar los paños de la fábrica tradicional. En ese caso se hace necesario construir paramentos exentos y ligeros, que sean compatibles con los movimientos del soporte y la heterogeneidad de su capacidad portante (8).
De este modo se pueden diseñar formatos integrados por piezas independientes, solidarias a tracción y normalmente ensambladas en seco. Esta nueva tipología de paramento demanda un desarrollo tecnológico del elemento cerámico y los elementos de unión, dado que éste se hace más complejo y de mayor alcance, para poder absorber mediante su propia sección gran parte de los requerimientos del sistema.

El valor añadido de la pieza cerámica, así como su capacidad vertebradora de los componentes aledaños le dan un fuerte protagonismo en la conformación del sistema y su apariencia final.

Esa evolución tipológica surge normalmente de la mano de las industrias cerámicas, dotadas de suficiente capacidad operativa y técnica para afrontar con solvencia los procesos de innovación necesarios para la fabricación de piezas cerámicas complejas. Esa disposición técnica ligada al material tiende hacia piezas de gran formato, minimizando la subestructura -normalmente metálica-, simplificando así sus conexiones y el montaje (9).

\subsubsection{Ejemplo B. Fachada ventilada con parteluz, call center para Telefónica Móviles en Toledo (2004)}

La sala principal de trabajo del edificio del call center de Toledo demanda cierta penumbra, luz no direccional, así como una gestión de las puntas térmicas (10) (11) (12).
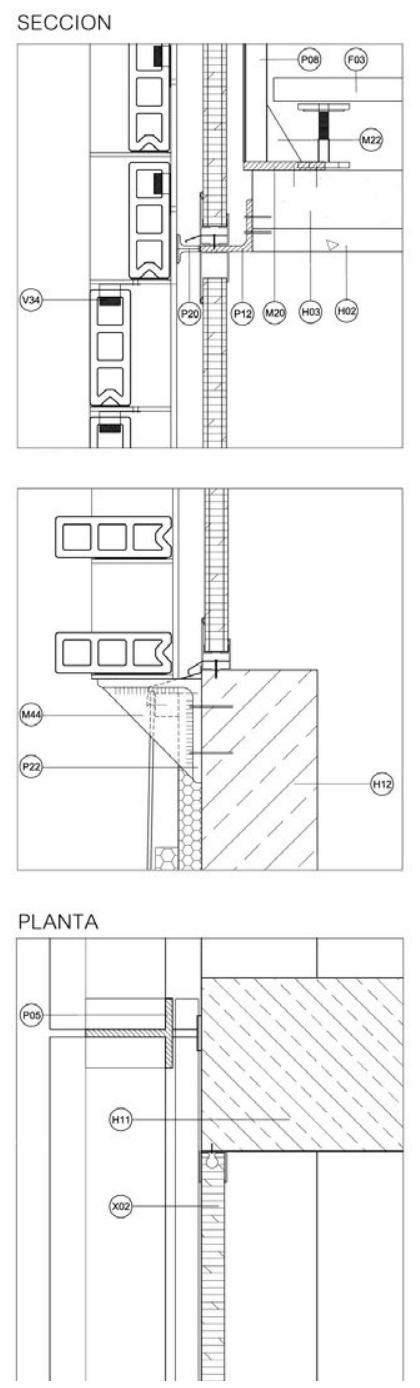

\begin{tabular}{|c|c|}
\hline & Perfiles \\
\hline P02 & Tubo $40 \times 80 \times 4$ \\
\hline P05 & T 140 \\
\hline P08 & $\mathrm{T} 40 \times 40$ \\
\hline P12 & L $90 \times 90 \times 10$ \\
\hline P18 & Tubo $80 \times 80 \times 4$ \\
\hline P19 & Tubo $140 \times 80 \times 5$ \\
\hline P20 & T 50, cortada \\
\hline \multirow[t]{2}{*}{ P22 } & L $180 \times 180 \times 15$ \\
\hline & Varios \\
\hline V13 & Anclaje para el X02 \\
\hline \multirow[t]{2}{*}{ V34 } & Anclaje mecánico con neopreno \\
\hline & Policarbonato \\
\hline $\mathrm{x} 02$ & Placa Lexa Thermoclick \\
\hline
\end{tabular}

Figura 3. Detalle de fachada.

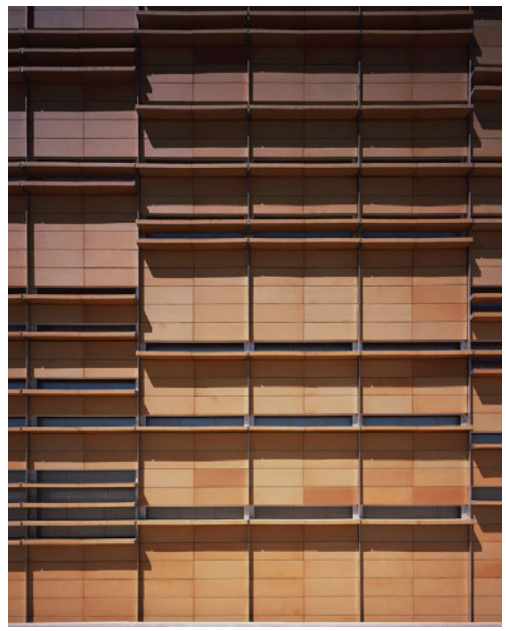

Figura 4. Detalle celosía.

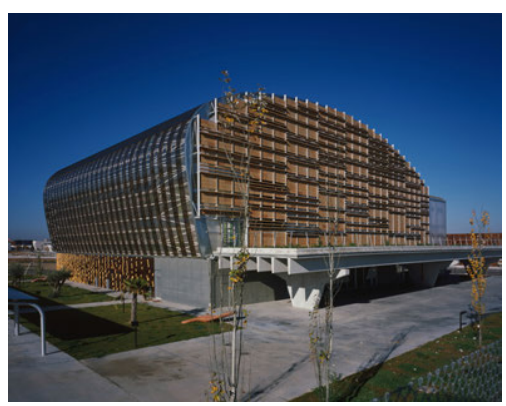

Figura 5. Paño celosía, fachada Este. 
A nivel tecnológico la pieza cerámica básica se fija dos a dos mediante la disposición de un pasador metálico por el alma y confinado por cuñas de neopreno. Este módulo, de 1,2 m de longitud resultante de la unión entre dos piezas cerámicas, es colgado a su vez de una subestructura de perfiles metálicos y conforma el conjunto del paramento. El esfuerzo de tener que solidarizar mecánicamente dos piezas cerámicas viene dado por la imposibilidad de obtener una planimetría del elemento cerámico sometido a temperaturas de cocción muy altas, y eso es debido, a su vez, a que las bajas temperaturas del invierno en Toledo precisan piezas de gran dureza y compacidad.

Finalmente, el paramento en cuestión desarrolla principalmente las propiedades térmicas de una fachada ventilada, con una filtración controlada de luz solar. El grado de perforación de la superficie cerámica es sólo del 30 \% y la luz que pasa al interior es difractada por una hoja de policarbonato celular. Además la pieza cerámica horizontal, desplegada siempre bajo cada abertura, refleja hacia el interior mayor intensidad de luz solar.

\subsubsection{Ejemplo C. Celosía Brise soley, vivienda unifamiliar} en Barcelona (2012)

En la obra de esta vivienda unifamiliar en Barcelona el paramento se dispone frente a una abertura completa de vidrio, actuando como modulador de la luz y la radiación solar. Siendo la estancia interior de carácter doméstico y reducido tamaño, es determinante el comportamiento térmico en su conjunto, maximizando la visión hacia el exterior (13).

Dado que la orientación de la superficie es de componente Sur, la separación entre lamas horizontales combinada con la anchura de cada pieza permite controlar la incidencia directa de los rayos de sol estacionalmente, de tal modo que en verano éstos no alcanzan el interior mientras que sí lo hacen masivamente en invierno. Así pues, la estancia interior puede calefactarse por efecto invernadero durante los meses de invierno (con una instalación complementaria de tubos de agua embebidos en el pavimento para gestionar las puntas térmicas durante el día y trasladarlas a la noche). En verano la verticalidad del rayo de sol rebota en la pieza cerámica

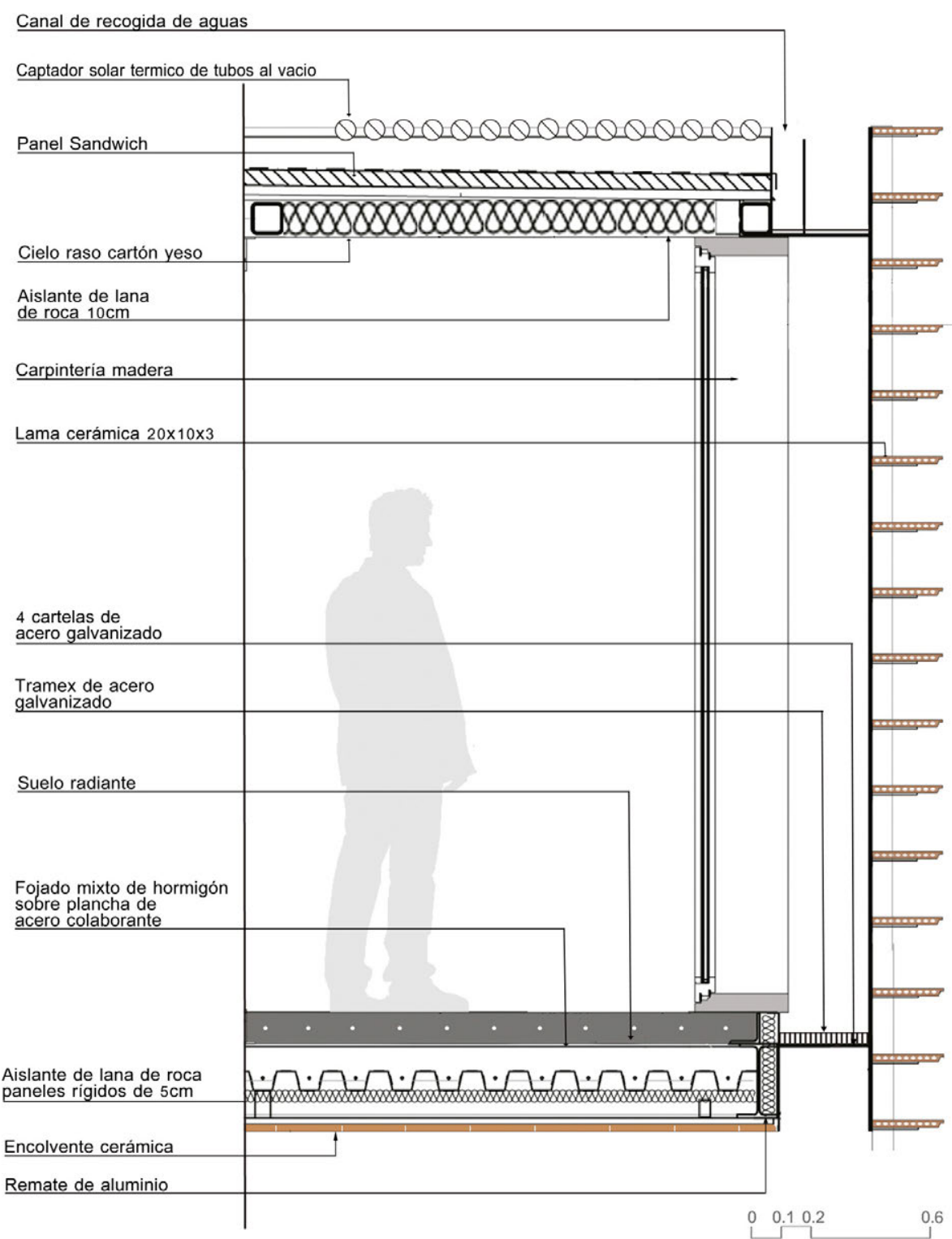

Figura 6. Sección constructiva.

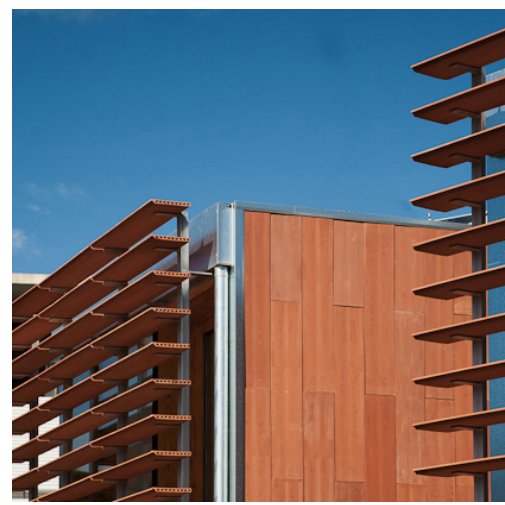

Figura 7. Detalle celosía.

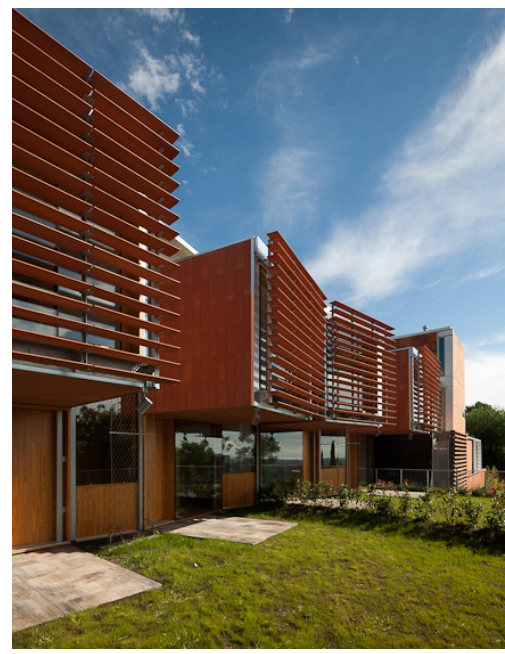

Figura 8. Vista conjunto. 
horizontal, de modo que la luz entra siempre reflejada sin calentar el interior.

También el calor irradiado por la pieza cerámica sobrecalentada por su exposición al sol no alcanza al interior al estar separada una distancia de $40 \mathrm{~cm}$ y tener libre convección de aire.

A nivel tecnológico la subestructura de soporte es metálica, a base de perfiles verticales cada 1,3 m, sobre los que se apoyan las piezas cerámicas que en un extremo están fijadas mediante resinas. A pesar de la amplitud del vano, éste se salva con una única pieza cerámica de canto muy fino. Esta pieza, obtenida por extrusión, tiene una planeidad perfecta en su perfil complejo, dado que para un clima meridional su fabricación no precisa de elevadas temperaturas de cocción del horno. Siendo el grado de perforación del sistema casi total, más que un paramento que neutraliza las condiciones exteriores, bien podemos hablar aquí de un filtro que las tamiza.

\subsection{De paramento a membrana}

La evolución de las construcciones hacia modelos más flexibles y ligeros, así como la necesidad de una construcción menos intensiva en mano de obra y material, sugiere el paso del paramento a la membrana cerámica. Es ésta una respuesta lógica en la transformación del sistema de celosía.

La membrana queda exenta de las solicitaciones que impone la rigidez, permitiendo el movimiento de todas y cada una de sus partes, lo que obliga de algún modo a reducir el tamaño de las piezas cerámicas para evitar excesiva torsión sobre ellas. Este aspecto conlleva cierta simplificación del componente cerámico propiamente dicho, en favor del engarce metálico que debido a las nuevas solicitaciones estructurales pasa a ser un integrante sustancial del sistema.
Las propiedades de la membrana aportan grandes ventajas para su industrialización, mediante el optimizado ensamblaje en fábrica de pequeñas piezas seriadas, conformando amplísimas superficies que viajarán plegadas para ser simplemente colgadas y tensadas en obra.

También el montaje permite elevados rendimientos de colocación, evitando al máximo el trabajo en andamiaje y concentrando las uniones en obra en muy pocos puntos y de fácil control.

La producción de membranas necesita cierto cambio estratégico por parte de los fabricantes de cerámica, al no focalizarse tanto en la producción óptima de piezas como en la confección e instalación de sistemas integrados.

Finalmente, este nuevo formato nos permite concebir amplias superficies cerámicas con menor implicación de material y fácil reciclabilidad de las partes, alineándose con los compromisos ambientales de nuestra sociedad contemporánea.

\subsubsection{Ejemplo D. Muro de confinamiento perimetral en ladera, Barcelona (2012)}

El sistema forma parte de un conjunto de muros de contención de tierras para el aterrazado del recinto de un jardín y su deslinde con la calle. El muro de contención es de hormigón armado, pero su exposición a la fuerte radiación solar de orientación SO, así como la ausencia de tierra a pie de muro, hacían imposible el crecimiento de vegetación sobre paramento exterior. La membrana cerámica tiene la doble función de sombrear la superficie exterior de hormigón y conformar un paramento resistente desde la calle, generando así una holgura atemperada propicia para el crecimiento de la vegetación (14) (15).

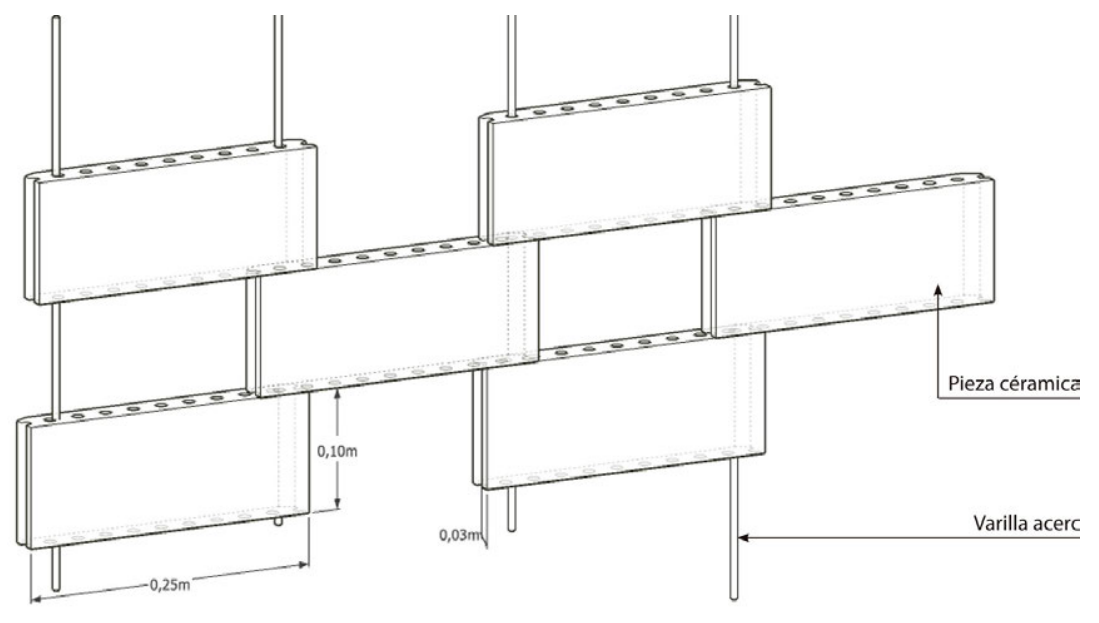

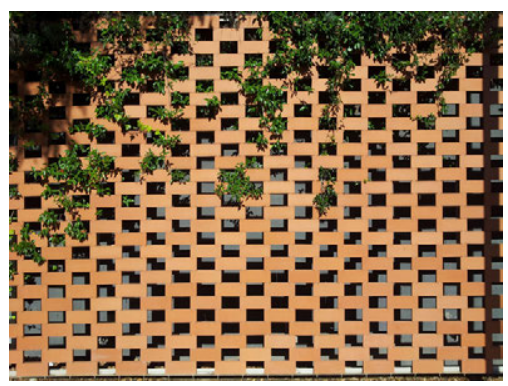

Figura 10. Vista.

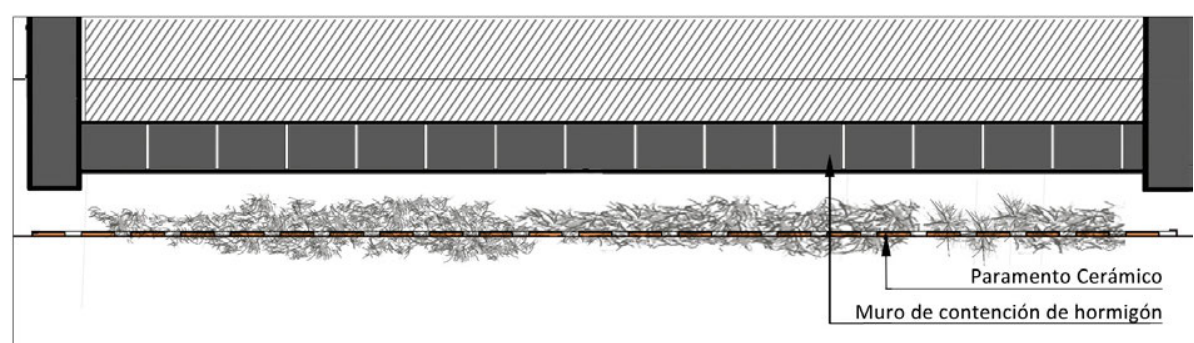

Figura 9. Detalle del sistema. 
El paramento tiene una perforación inferior al 40\%, produciendo un denso sombreamiento de la cámara posterior, que con apenas $30 \mathrm{~cm}$ de grosor cuenta a su vez con la aportación térmica del terreno (entorno a los $18^{\circ} \mathrm{C}$ ) a través del muro inercial de hormigón. Esas condiciones de penumbra atemperada son especialmente adecuadas para el desarrollo y floración de ciertas especies de vegetación como el jazmín, que en ningún caso habría podido desarrollarse libremente.

A nivel tecnológico la superficie exterior está formada por una única tipología de pieza cerámica extruida, de pequeño formato y perfil sencillo. Las piezas cerámicas son engarzadas a través de su alma por varilladas verticales de acero, fijadas a un marco perimetral para conformar un paramento autoportante. La superficie se asemeja a una membrana tejida, con trama cerámica y urdimbre de acero, permitiendo el libre movimiento de sus partes en respuesta al impacto y la dilatación.

Finalmente, los paramentos fueron montados enteramente en fábrica y simplemente fijados al muro de hormigón. El coste del transporte supone una repercusión menor, al tratarse de unidades bidimensionales apilables.

\subsubsection{Ejemplo E. Celosía para un aulario escolar, Barcelona (2014)}

Se analiza la fachada urbana del nuevo aulario para las escuelas teresianas de Barcelona (16). Se trata de una edificación contemporánea dentro de un recinto histórico-artístico, cuyo edificio fundacional es obra de Antoni Gaudí. El contexto viene determinado por la fuerte presencia de los edificios históricos, con su tradicional fábrica de ladrillo ejecutada en un momento de máximo despliegue técnico y expresivo de los oficios tradicionales. En su alineación con la calle el volumen presenta una superficie cerámica, mostrando de algún modo hacia la cuidad la traza del material que identifica el recinto.

La celosía esta encarada a S-O, tamiza la fuerte incidencia solar en verano frente a dos ámbitos distintos en su trasdós. Por un lado está la caja de escalera y circulaciones verticales del edificio, delimitada por una capa de policarbonato celular que difracta la luz directa y proporciona estanqueidad. La separación entre la capa cerámica y el policarbonato induce un flujo de aire que disipa el calor capturado en la pieza cerámica por radiación solar. A continuación la celosía se extiende sobre un espacio tampón abierto de $1 \mathrm{~m}$ de anchura, con jardineras lineales de tipo hidropónico formando

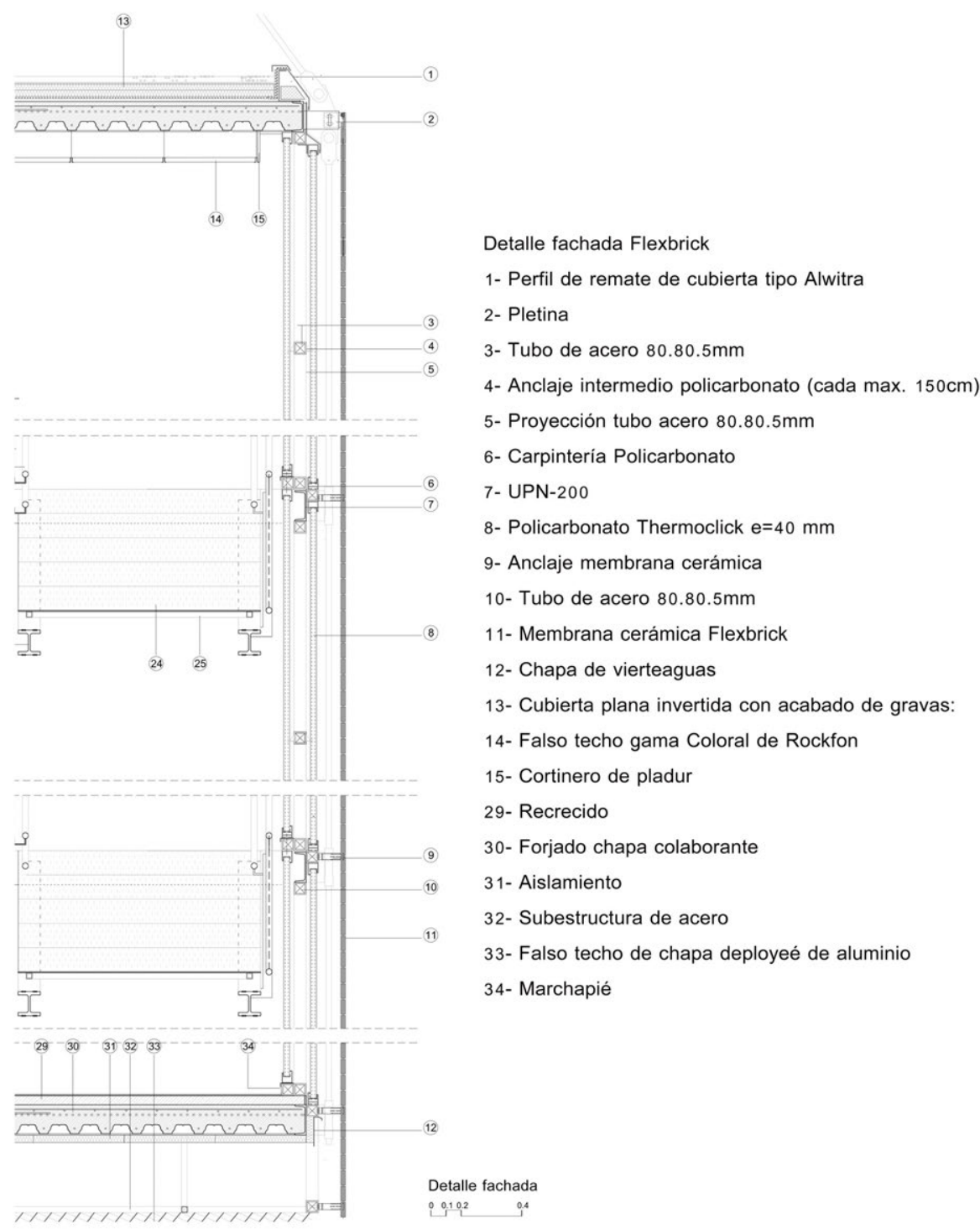

Figura 11. Detalle de fachada.

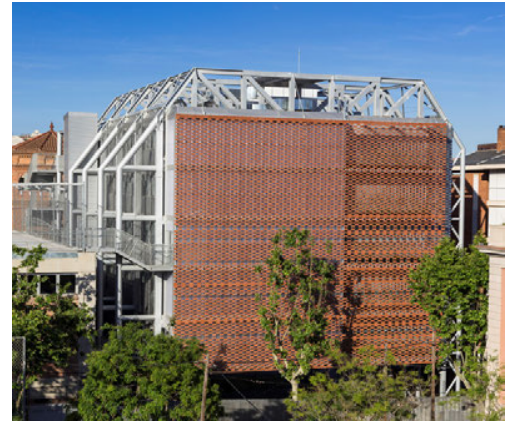

Figura 12. Vista del conjunto.

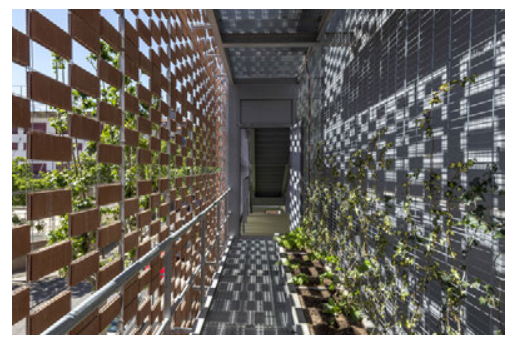

Figura 13. Vista interior con jardín vertical. 


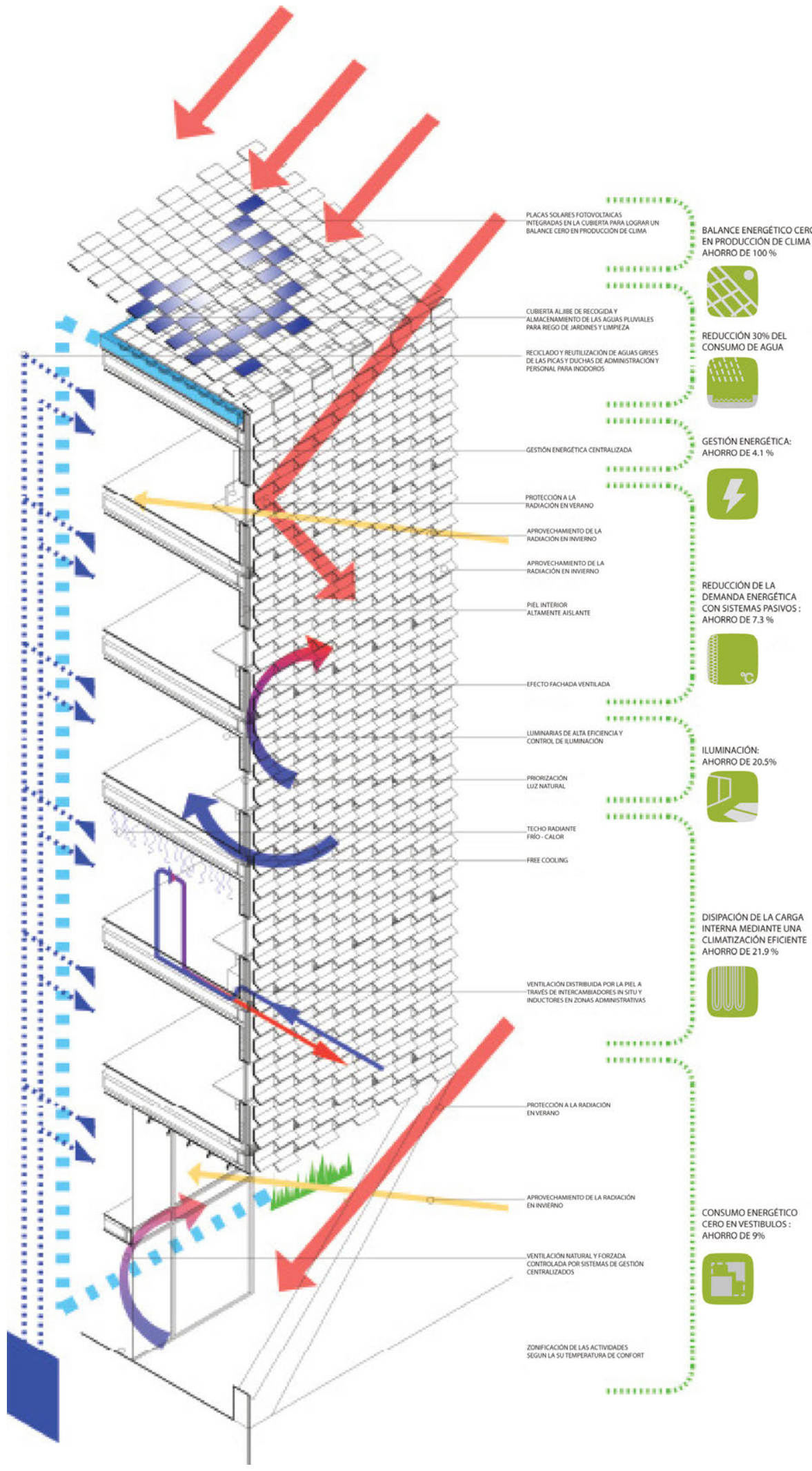

Figura 14. Axonometría de detalle del sistema de envolvente.

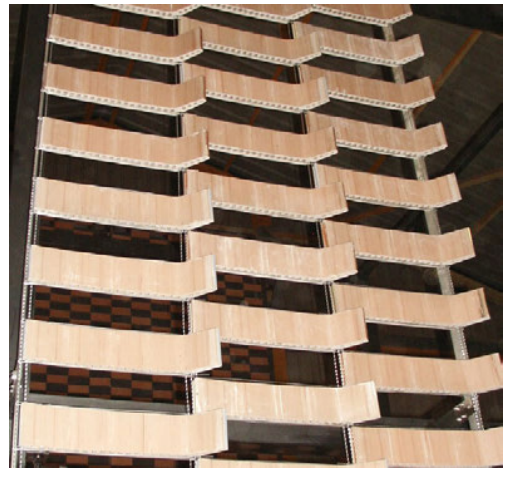

Figura 15. Prototipo de celosía. Vista exterior.

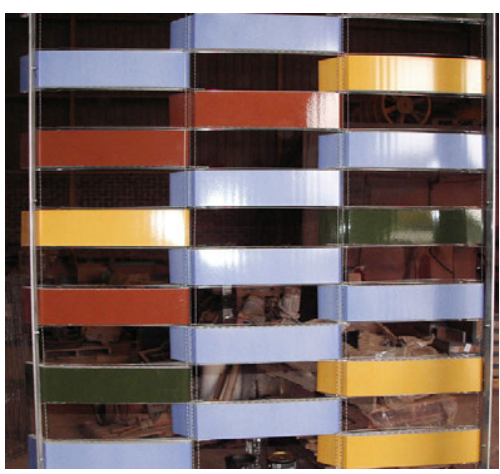

Figura 16. Prototipo de celosía. Vista interior. un jardín vertical. Las condiciones de penumbra favorecen el desarrollo de la vegetación trepadora, estableciendo un microclima atemperado, que actúa como colchón térmico frente al espacio de aulas colindante.

A nivel tecnológico la celosía cerámica es literalmente una membrana compuesta por la agregación de una única pieza extruida de terracota, engarzada a través de una malla de acero trenzado según modelo del prototipo industrial «Flexbrick». Tramos de celosía a toda altura $(12 \times 1,2 \mathrm{~m})$ son transportadas a obra en rollos para ser colgadas, desplegadas y tensadas, enlazándose en una única superficie continua mediante perfiles de acero que les atan lateralmente y anclan a la estructura principal, limitando de ese modo deformaciones 
excesivas en caso de fuerte viento. El calado tiene una opacidad del 50\% y arroja la sombra directa de su patrón plano.

\subsection{Membranas más tridimensionales}

Llegados a este punto, podemos conjeturar algunos caminos futuros para este sistema constructivo, teniendo en cuenta la capacidad de sus prestaciones para ir respondiendo a las aspiraciones propias de nuestro tiempo.

En ese sentido, la recuperación de cierta tridimensionalidad en la membrana permitiría sacar mayor partido de sus condiciones de filtro, muy especialmente en cuanto a la protección y refracción de la luz solar, e incluso la producción energética de origen solar.

Ese nuevo estadio de evolución precisa, sin embargo, despejar algunos interrogantes, cuya resolución puede abrir diversos frentes de desarrollo. La recuperación de la tridimensionalidad lleva implícita una mayor complejidad de la pieza cerámica que articula el sistema y abre un vasto camino de exploración. Esa circunstancia deja en manos de la industria cerámica, de su capacidad y empuje, la posibilidad de liderar su desarrollo futuro.

\subsubsection{Ejemplo F. Envolvente para edificio de laboratorios,} Barcelona. Obra en curso (2016)

Se describe la envolvente continua proyectada para el nuevo centro de investigación médica del hospital San Pau de Barcelona y cuya obra se halla aún en curso. La propuesta cerámica resuelve la totalidad de la envolvente del edificio, respondiendo a todas y cada una de sus caras expuestas -fachadas $\mathrm{N}, \mathrm{S}$, E, O, cubierta y trasdós del porche de la planta baja.

La envolvente de cubierta genera un espacio sombreado por encima de una cubierta aljibe, creando un espacio frondoso y confortable, que actúa a su vez como tampón térmico. Esa envolvente de cubierta incorpora sistemáticamente tecnología para la producción solar térmica y fotovoltaica. Las distintas orientaciones de las fachadas verticales se resuelven a partir de una familia de piezas tridimensional, cuya disposición en cada cara viene condicionada por la direccionalidad de la luz solar. Las piezas cuentan con una cara vidriada que intensifica la reflexión de los rayos solares, creando de este modo cierta reverberación lumínica entre el cerramiento y la membrana.

A nivel tecnológico el sistema retoma el modelo industrial «Flexbrick», redimensionando el alcance de sus partes tratando de dar mayores alcances a las prestaciones para este caso concreto. El tamaño de la pieza cerámica, así como su componente tridimensional y el vidriado de la cara interior, suponen una mayor complejidad del elemento, frente al engarce metálico que sigue siendo estándar. La tridimensionalidad de la pieza cerámica permite combinar un notable grado de transparencia (50\%) con una completa protección solar.

\section{CUADRO COMPARATIVO DE SISTEMAS}

A modo de resumen comparativo se introducen los parámetros de cada uno de los sistemas.

\begin{tabular}{|c|c|c|c|c|c|c|}
\hline & A. (Panamá) & B. (Toledo) & C. (Pomaret 1) & D. (Pomaret 2) & E. (Teresianas) & F. (St. Pau) \\
\hline $\begin{array}{l}\text { Sistema } \\
\text { constructivo }\end{array}$ & Celosía & $\begin{array}{l}\text { Fachada ventilada } \\
\text { por parteluz }\end{array}$ & Fachada de lamas & $\begin{array}{l}\text { Celosía como muro } \\
\text { perimetral }\end{array}$ & Tejido cerámico & Tejido cerámico 3D \\
\hline $\begin{array}{l}\text { Grado de } \\
\text { personalización }\end{array}$ & $\begin{array}{l}\text { Múltiples según } \\
\text { diseño y pericia del } \\
\text { operario }\end{array}$ & $\begin{array}{l}\text { Diferentes } \\
\text { disposiciones de una } \\
\text { pieza estándar }\end{array}$ & $\begin{array}{l}\text { Elaboración de una } \\
\text { pieza cerámica de } \\
\text { medida }\end{array}$ & Pieza estándar & Pieza estándar & $\begin{array}{l}\text { Elaboración de } 2 \text { piezas } \\
\text { cerámicas realizadas a } \\
\text { medida, que rotan en } \\
\text { relación a la orientación }\end{array}$ \\
\hline Geometría & $\begin{array}{l}\text { Paralepípedo en } \\
\text { masa }\end{array}$ & $\begin{array}{l}\text { Paralepípedo } \\
\text { aligerado }\end{array}$ & $\begin{array}{l}\text { Paralepípedo } \\
\text { aligerado }\end{array}$ & $\begin{array}{l}\text { Paralepípedo } \\
\text { aligerado }\end{array}$ & Paralepípedo aligerado & $\begin{array}{l}\text { Paralepípedo aligerado, } \\
\text { pieza en L aligerada }\end{array}$ \\
\hline $\begin{array}{l}\text { Dimensiones de } \\
\text { la pieza cerámica } \\
(\mathrm{mm})\end{array}$ & $290 \times 140 \times 50 \mathrm{~mm}$ & $1.400 \times 200 \times 70 \mathrm{~mm}$ & $910 \times 200 \times 30 \mathrm{~mm}$ & $239 \times 97 \times 30 \mathrm{~mm}$ & $239 \times 97 \times 30 \mathrm{~mm}$ & $\begin{array}{l}96 \times 409 \times 30 \\
96 \times 386 \times 30(\mathrm{~L}) \\
85 \times 409 \times 30(\mathrm{~L}) \mathrm{mm} \\
\end{array}$ \\
\hline $\begin{array}{l}\text { Masa de la pieza } \\
\text { cerámica }\end{array}$ & $4,06 \mathrm{~kg}$ & $16,8 \mathrm{~kg}$ & $10,92 \mathrm{~kg}$ & $1,22 \mathrm{~kg}$ & $1,22 \mathrm{~kg}$ & $1,79 \mathrm{~kg} ; 2,13 \mathrm{~kg} ; 3,12 \mathrm{~kg}$ \\
\hline $\begin{array}{l}\text { Fabricante de la } \\
\text { pieza cerámica }\end{array}$ & Desconocido & Cerámicas La Paloma & LCM Lacer España & Flexbrick & Flexbrick & Flexbrick \\
\hline $\begin{array}{l}\text { Relación } \\
\text { lleno/vacío de la } \\
\text { fachada }\end{array}$ & $40 \%$ & $89 \%$ & $30 \%$ & $50 \%$ & $50 \%$ & $50 \%$ \\
\hline $\begin{array}{l}\text { Tecnología de } \\
\text { fijación }\end{array}$ & $\begin{array}{l}\text { Pieza a pieza } \\
\text { amorteradas }\end{array}$ & $\begin{array}{l}\text { Pieza a pieza sobre } \\
\text { estructura metálica }+ \\
\text { alma interior metálica }\end{array}$ & $\begin{array}{l}\text { Pieza a pieza sobre } \\
\text { estructura metálica }\end{array}$ & $\begin{array}{l}\text { Paño premontado } \\
\text { ensamblado a } \\
\text { varillas metálicas }\end{array}$ & $\begin{array}{l}\text { Mantas pre-ensambladas } \\
\text { de } 1,20 \times 12,00 \mathrm{~m} \text {, colgadas } \\
\text { a estructura metálica }\end{array}$ & $\begin{array}{l}\text { Mantas pre-ensambladas } \\
\text { de } 1,25 \text { ancho y longitud } \\
\text { variable, colgados a } \\
\text { estructura metálica }\end{array}$ \\
\hline $\begin{array}{l}\text { Disposición en } \\
\text { fachada en relación } \\
\text { al sol }\end{array}$ & $\begin{array}{l}\text { Celosía. } \\
\text { Orientación Sur }\end{array}$ & $\begin{array}{l}\text { Pared opaca con } \\
\text { lama, orientaciones } \\
\text { Este y Oeste }\end{array}$ & $\begin{array}{l}\text { Pared de lamas, } \\
\text { orientación Sur }\end{array}$ & $\begin{array}{l}\text { Celosía en damero, } \\
\text { orientación Sur }\end{array}$ & $\begin{array}{l}\text { Membrana permeable, } \\
\text { orientación Suroeste }\end{array}$ & $\begin{array}{l}\text { Membrana permeable, } \\
\text { acabado mate al exterior y } \\
\text { cara refrectante al interior, } \\
\text { todas las orientaciones }\end{array}$ \\
\hline $\begin{array}{l}\text { Tipo de paramento } \\
\text { interior/Distancia } \\
\text { entre paramentos }\end{array}$ & Vidrio/50 cm & $\begin{array}{l}\text { Policarbonato } \\
\text { celular } / 12 \mathrm{~cm}\end{array}$ & Vidrio $/ 40 \mathrm{~cm}$ & $\begin{array}{l}\text { Muro de } \\
\text { hormigón/20 cm }\end{array}$ & $\begin{array}{l}\text { Policarbonato } \\
\text { celular } / 100 \mathrm{~cm}\end{array}$ & $\begin{array}{l}\text { Panel sándwich, } \\
\text { vidrio } / 30 \mathrm{~cm}\end{array}$ \\
\hline Acabado/Color & Ninguno/Térreo & Ninguno/Térreo & Ninguno/Térreo & Ninguno/Térreo & Ninguno/Térreo & $\begin{array}{l}\text { Esmaltado por la cara } \\
\text { interior/Amarillo, verde, } \\
\text { azul, térreo }\end{array}$ \\
\hline $\begin{array}{l}\text { Distancia entre } \\
\text { producción } \\
\text { e instalación }\end{array}$ & - & $37 \mathrm{~km}$ & $353 \mathrm{~km}$ & $42 \mathrm{~km}$ & $42 \mathrm{~km}$ & $51 \mathrm{~km}$ \\
\hline
\end{tabular}

Figura 17. Cuadro comparativo de características de cada sistema de celosía cerámica. 


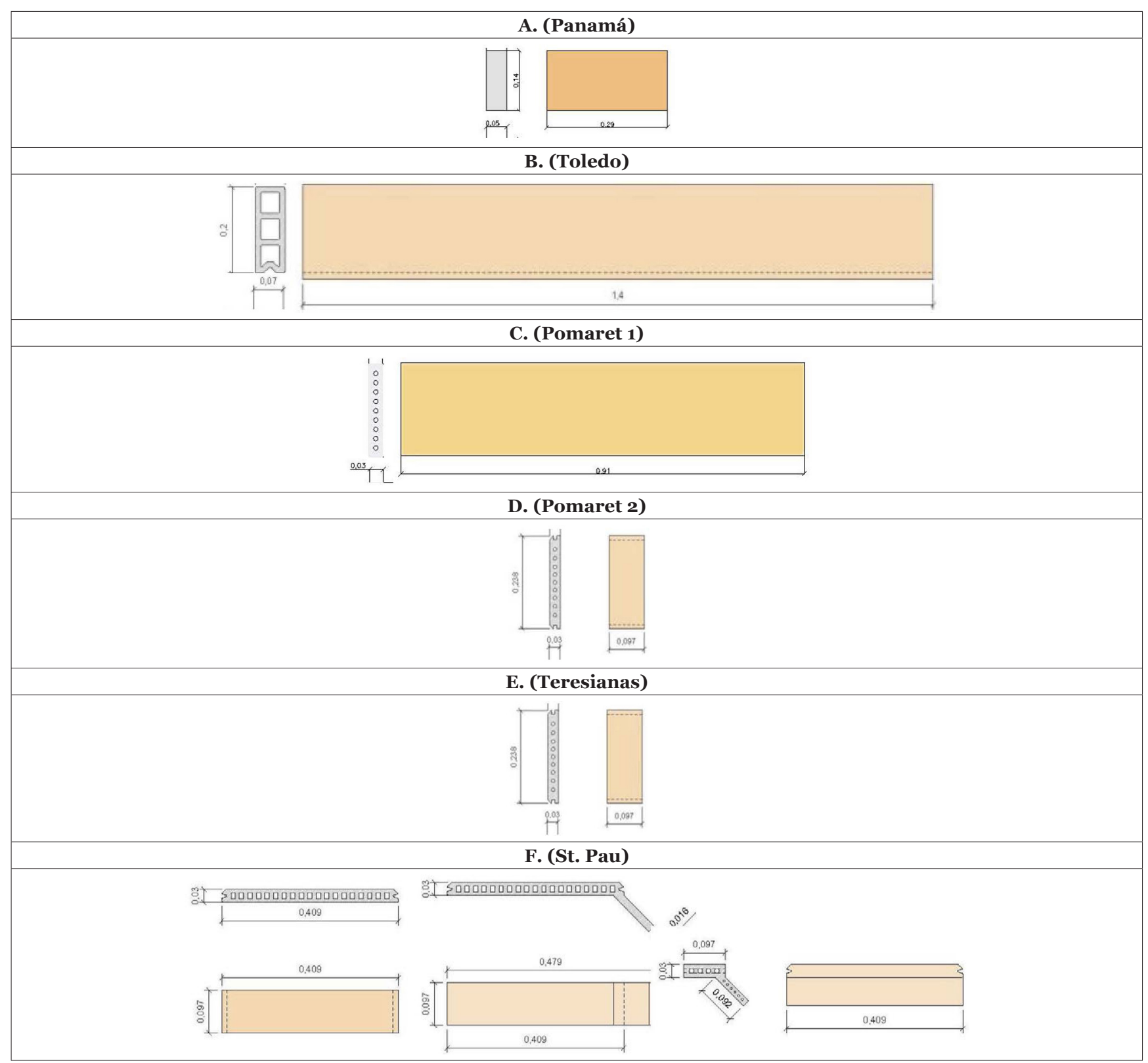

Figura 18. Cuadro comparativo de las geometrías de las piezas cerámicas.

\section{CONCLUSIONES}

Mediante el análisis comparativo de 5 soluciones de filtros solares cerámicos, los autores se atreven a apuntar que:

1. Las celosías cerámicas tienden a evolucionar tecnológicamente hacia la ligereza, la flexibilidad de colocación y el control de ejecución, siendo una respuesta idónea a re- querimientos energéticos de la edificación, garantizando el control de la radiación solar y de la demanda energética (17).

2. El tejido industrial de la cerámica es capaz de dar respuesta a un desarrollo tecnológico que se vertebra a través de piezas cerámicas cada vez más complejas que pueden constituir sistemas abiertos, maximizando sus aportaciones físicas y ambientales en la arquitectura (18).

\section{REFERENCIAS}

(1) Paricio, I. El hueco en la fachada. Tectónica: Monografías de Arquitectura, Tecnología y Construcción (4). ISSN: 11360062.

(2) Puigdomènech Franquesa, J., Cusidó Fábregas, J.A., Porta i Jué, J. (1986). Ahorro energético mediante estrategias de iluminación natural optimizadas. Informes de la Construcción, 37(379): 33-41. http://dx.doi.org/10.3989/ic.1986.v37. i379.1771.

(3) Larrumbide, E., Bedoya, C. (2015). El comportamiento del hueco de ventana en la arquitectura vernácula mediterránea española ante las necesidades de acondicionamiento solar. Informes de la Construcción, 67(539): e105. http://dx.doi. org/10.3989/ic.14.056. 
(4) Cusidó, J.A., Puigdomènech, J. (1984). Dimensionado de controles solares fijos en función de la aportación de radiación solar directa y de la orientación de la pared. Informes de la Construcción, 36(364): 41-54. http://dx.doi.org/10.3989/ ic.1984.v36.i364.1906.

(5) Toplicic-Curcic, G., Grdic, D., Ristic, N., Grdic, Z. (2015). Ceramic façade cladding as an element of sustainable development UDC 502.131.1. FACTA UNIVERSITATIS Series: Architecture and Civil Engineering, 13(3): 219-231.

(6) Salas, J. (2008). De los sistemas de prefabricación cerrada a la industrialización sutil de la edificación: algunas claves del cambio tecnológico. Informes de la Construcción, 6o(512): 19-34. http://dx.doi.org/10.3989/ic.07.001.

(7) Yáñez Parareda, G. (1982). Energía solar edificación y clima, tomos I y II. Madrid: Ministerio de Obras Públicas y Urbanismo.

(8) Pich-Aguilera, F., Batlle, T., Sauer, B. (2001). La arcilla tecnológica. Nuevo origen para la arquitectura contemporánea. Actar. ISBN: 84-95273-84-5.

(9) Minguet, J.M., Coste, A., Mestre, O., Maurette, C. (2013). Slat façades. Fachadas en celosía. Instituto Monsa de Ediciones. ISBN: 978-84-15829-23-2.

(10) Pich-Aguilera, F., Batlle, T. (2007). Edificio de la sede de Telefónica en Toledo. Techniques \& Architecture (492): 64-67. ISSN: 0373-0719.

(11) Pich-Aguilera, F., Batlle, T. (2006). Edificio para Telefonía móvil en Toledo. Temas de Arquitectura, 1 (4): 140-155. ISSN: 1698-8671.

(12) Pich-Aguilera, F., Batlle, T. (2006). Call Center Toledo. ConArquitectura (18). ISSN: 1578-0201.

(13) Pich-Aguilera, F. (2006). Etiqueta Verde. Arquitectura Viva (105). ISSN: 0214-1256.

(14) Sarrablo, V. (2008). Cortezas Cerámicas. Caleidoscópio-Ediçao e Artes Gráficas, S. A. ISBN: 978-989-8129-54-3.

(15) Pich-Aguilera, F., Batlle, T. (2013). Viviendas pareadas «Pomaret». ConArquitectura (48): 35-42. ISSN: $1578-0201$.

(16) Pich-Aguilera, F., Batlle, T. (2014). Ampliación del colegio «Teresiana-Ganduixer». ConArquitectura (52): 14-21. ISSN: 1578-0201.

(17) Pich-Aguilera, F. (2005). Arquitectura industrializada, una respuesta real (pp. 1-71). Actar. ISBN: 8460943194.

(18) Russo, M., Börkey, P., Cubel, E., Lévêque, F., Mas, F. (1996). Local Sustainability and Competitiveness: The Case of the Ceramic Tile Industry. Materiali di discussion (134). European Foundation for the Improvement of Living and Working Conditions. 